\title{
THE ACCELERATING EXPANSION OF THE UNIVERSE AND TORSION ENERGY
}

\author{
M.I.WANAS \\ Astronomy Department, Faculty of Science, Cairo University, Giza, Egypt. \\ E-mail:wanas@mailer.eun.eg
}

\begin{abstract}
In the present work, it is shown that the problem of the accelerating expansion of the Universe can be directly solved by applying Einstein geometrization philosophy in a wider geometry. The geometric structure used to fulfil the aim of the work is a version of Absolute Parallelism geometry in which curvature and torsion are simultaneously non vanishing objects. It is shown that, while the energy corresponding to the curvature of space- time gives rise to an attractive force, the energy corresponding to the torsion indicates the presence of a repulsive force. A fine tuning parameter can be adjusted to give the observed phenomena.

Keywords: Dark Energy, Torsion, Anti-Gravity.

PACS numbers: 98.80.-K, 95.36.+X, 79.60.BW.
\end{abstract}

\section{Introduction}

Recently, observation of red-shift of type $I a$ supernovae $^{1}$ indicate that our Universe is in an accelerating expansion phase. The four known fundamental interactions, as we understand them today, can not account for the accelerating expansion of the universe. Weak and strong interactions can be easily ruled out, since both are very short range and cannot play any role in the large scale behavior of our Universe. Also, electromagnetism cannot play any role, in this respect, since neither the Universe nor its material constituents are electrically charged, while monopoles are not present in our Universe. The only interaction which is playing the main role, in the structure and evolution of the universe, is gravity. But gravity, as far as we know, is attractive and not repulsive. Consequently, it can not give any satisfactory interpretation of the accelerating phase of the Universe. One can deduce that, either gravity is not well understood and an important ingredient is missing in gravity theories, or a new interaction is about to be discovered, in order to account for such observations.

Gravity theories are usually constructed to give a better understanding of gravitational interactions, from both quantitative and qualitative points of view. Newton's theory deals with gravity as a force acting at a distance. This theory has suffered from some problems when applied to the motion in the solar system (the advance of perihelion of Mercury's orbit). In addition, the theory has shown its non-invariance under Lorentz transformations. These problems motivated Einstein to construct a new theory for gravity, the "General theory of Relativity" (GR). In addition to the solution of the problems of Newton's theory, GR gives very successful interpretation of gravitational phenomena in the solar system, binary star system and many other systems in the Universe. Also, GR has predicted the existence of a number of phenomenae which has been confirmed observationally, afterwards. Both Newton's theory and Einstein's theory give rise to an 
attractive force. So, neither of these theories, in their orthodox forms, can account for supernovae type Ia observations, since this needs the existence of repulsive force which is missing in both theories.

Now since the problem of SN type Ia observation has no satisfactory solution and is still challenging, one cannot reject any assumption which may lead to a solution. Among these assumptions are:

(a) The existing physics is not adequate for interpreting all known phenomena in our Universe. One may need new physics which would imply new interactions.

(b) The existence of a new type of matter in the Universe, with new properties, that can lead to the observed accelerating expansion of the Universe.

(c) One or more of the known fundamental interactions are not well understood and need further investigation. In particular, there may be a gap in our present

understanding of gravity ${ }^{1}$.

Many authors, in order to solve the above mentioned problem, have returned back to a modified version of GR, in which the cosmological constant existed in the theory (see Ref. 2).Although this constant can solve the problem, since it may give rise to a repulsive force, it suffers from a big problem the " cosmological constant problem" ${ }^{3}$. However, GR still has its attractive feature i.e. its philosophical basis, the "geometrization philosophy". This philosophy still deserves further investigations. It may provide solutions to gravity problems especially those connected to SN observations.

The aim of the present work is to re-examine the geometrization philosophy, seeking a solution for gravity problems. In the next section, a brief review of the geometerization philosophy is given. In section 3, a summary of the geometric structure, used in the the present work, is given. Section 4 gives an attempt to solve the problem addressed above using the torsion energy. The work is discussed and concluded in section 5 .

\section{Summary of the Geometrization Philosophy}

In constructing his theory of GR, Einstein has invented an ingenious idea that geometry can be used to solve physical problems. This idea is known as the "Geometrization of Physics". It comprises a philosophical principle which can be summarized in the following statement.

"To understand Nature one has to start with Geometry and end with Physics".

Einstein has applied this philosophy using the following guide lines:

1. Laws of nature are identities in the chosen geometry.

2. There is a one-to-one correspondence between physical quantities and geometric objects.

3. Physical trajectories of test particles are geometric paths (curves) in the chosen geometry.

Einstein has chosen the 4-dimensional Riemannian geometry, with the following identifi-

cations corresponding to the 3 -guide lines given above ${ }^{4}$, to represent the physical world including space and time: 
1. Conservation, as a law of nature, corresponds to the second contracted Bianchi identity,

$$
\left(R^{\mu \nu}-\frac{1}{2} g^{\mu \nu} R\right)_{; \nu} \equiv 0
$$

where $R^{\mu \nu}$ is Ricci tensor, $R$ is Ricci scalar and the semicolon is used for covariant differentiation.

2. The conserved quantity (the quantity between brackets),

$$
G^{\mu \nu} \stackrel{\text { def }}{=} R^{\mu \nu}-\frac{1}{2} g^{\mu \nu} R,
$$

is constructed from the curvature tensor (built using Christoffel symbol). This quantity corresponds to a type of energy that causes the curvature of the space. (For this reason, geometrically speaking, in what follows we are going to call the quantity defined by (2) "The Curvature Energy").

3. Taking the third guide line into consideration, Einstein has used the geodesic equation

$$
\frac{d^{2} x^{\mu}}{d s^{2}}+\left\{\begin{array}{c}
\mu \\
\alpha \beta
\end{array}\right\} \frac{d x^{\alpha}}{d s} \frac{d x^{\beta}}{d s}=0,
$$

to represent the trajectory of any test particle in the field and the null-geodesic equation,

$$
\frac{d^{2} x^{\mu}}{d \lambda^{2}}+\left\{\begin{array}{c}
\mu \\
\alpha \beta
\end{array}\right\} \frac{d x^{\mu}}{d \lambda} \frac{d x^{\nu}}{d \lambda}=0,
$$

to represent the trajectory of photons.

On the above scheme, we have the following comments:

1. The choice of Riemannian geometry represents a limited case, since this geometry has a vanishing torsion. This choice would not give a complete description of the physical world including space-time. Einstein has realized this fact in his subsequent attempts to construct unified field theories ${ }^{4,5}$ and has used geometries with torsion in these attempts.

2. The use of equation (3)or (4) to describe motion of test particles implies the application of the equivalence principle. This may represent a desirable feature in describing the motion of a scalar test particle (particle defined completely by its mass (energy)). Note that, most of elementary constituents of the Universe are fermions (particles with mass, spin, charge, .... ), for which (3) and (4) are no longer applicable.

3. The most successful results of GR, which confirm the theory, are those obtained using the field equations for empty space (pure geometric). The use of the full field equations of GR (equations containing a phenomenological matter tensor) is almost problematic.

Considering these comments, we are going to examine the application of the geometrization philosophy to geometries with simultaneously non-vanishing curvature and torsion. 


\section{Geometries with Curvature and Torsion}

Torsion tensor is the antisymmetric part of any non-symmetric linear connection. Einstein has used two types of geometries, with non-vanishing torsion, in his attempts unify gravity and electromagnetism. The first type is the Absolute Parallelism (AP) geometry with non vanishing torsion but vanishing curvature ${ }^{5}$. The second is of Riemann-Cartan type with simultaneously non-vanishing torsion and curvature ${ }^{4}$. In what follows, we are going to review very briefly a version of AP-geometry in which both torsion and curvature are simultaneously non-vanishing. We have chosen this type since calculations in its context are very easy.

The structure of a 4-dimensional AP-space is defined completely by a set of 4-contravariant linearly independent vector fields $\underset{i}{\lambda^{\mu}}$. The covariant components of such vector fields are defined such that (for further details see Ref. 6),

$$
\begin{aligned}
& { }_{i}^{\lambda^{\mu}}{ }_{i}{ }_{\nu}=\delta_{\nu}^{\mu}, \\
& \lambda_{i}^{\lambda^{\mu}}{ }_{j}{ }_{\mu}=\delta_{i j}
\end{aligned}
$$

The second order symmetric tensors,

$$
\begin{aligned}
& g_{\mu \nu} \stackrel{\text { def }}{=} \underset{i}{\lambda_{\mu}} \underset{i}{\lambda_{\nu}}, \\
& g^{\mu \nu} \stackrel{\text { def }}{=} \underset{i}{\lambda_{i}^{\mu}} \underset{i}{\lambda^{\nu}},
\end{aligned}
$$

can play the role of the metric tensor, and its conjugate, of the Riemannian space associated with the AP-space. With the help of (7) and (8) one can define a linear connection (Christoffel symbol of the second kind), using which one can perform covariant derivatives, as usual. Another linear connection can be obtained as a consequence of AP-condition,

$$
\lambda_{i+\mid \nu}^{\lambda \mu}=0
$$

This equation can be solved to give the following non-symmetric linear connection $\Gamma_{. \mu \nu}^{\alpha}$ (the $(+)$ sign is used to characterize covariant derivative using this connection),

$$
\Gamma_{. \mu \nu}^{\alpha} \stackrel{\text { def }}{=} \lambda_{i}^{\alpha}{ }_{i} \lambda_{\mu, \nu}=\left\{\begin{array}{c}
\alpha \\
\mu \nu
\end{array}\right\}+\gamma_{. \mu \nu}^{\alpha},
$$

where $\left\{\begin{array}{c}\alpha \\ \mu \nu\end{array}\right\}$ is Christoffel symbol of second the second kind and $\gamma_{. \mu \nu}^{\alpha}$ is the contortion tensor, given by

$$
\gamma_{. \mu \nu}^{\alpha} \stackrel{\text { def }}{=} \lambda_{i}^{\alpha}{ }_{i} \lambda_{\mu ; \nu}
$$

the semicolon is used to characterize covariant differentiation using Christoffel symbol. Now the torsion of the AP-space is defined by

$$
\Lambda_{. \mu \nu}^{\alpha}=\Gamma_{. \mu \nu}^{\alpha}-\Gamma_{. \nu \mu}^{\alpha}=\gamma_{. \mu \nu}^{\alpha}-\gamma_{. \nu \mu}^{\alpha}
$$


The relation between (11) and (12) can be written as ${ }^{7}$,

$$
\gamma_{\mu \nu}^{\alpha}=\frac{1}{2}\left(\Lambda_{. \mu \nu}^{\alpha}-\Lambda_{\mu . \nu}^{\alpha}-\Lambda_{\nu . \mu}^{\alpha}\right)
$$

The relations(12) and (13) implies that the vanishing of the torsion is a necessary and sufficient condition for the vanishing of the contortion. The curvature tensor corresponding to the linear connection (10) can be written, in the usual manner, as

$$
B_{\mu \nu \sigma}^{\alpha} \stackrel{\text { def }}{=} \Gamma_{\mu \sigma, \nu}^{\alpha}-\Gamma_{\mu \nu, \sigma}^{\alpha}+\Gamma_{\mu \sigma}^{\epsilon} \Gamma_{\epsilon \nu}^{\alpha}-\Gamma_{\mu \nu}^{\epsilon} \Gamma_{\epsilon \sigma}^{\alpha} .
$$

This tensor vanishes identically because of (9). Although this appears as a disappointing feature, but we are going, in the next section, to show that it represents a cornerstone in the present work. The curvature tensor (14) can be written in the form,

$$
B_{\mu \nu \sigma}^{\alpha}=R_{\mu \nu \sigma}^{\alpha}+Q_{\mu \nu \sigma}^{\alpha} \equiv 0,
$$

where,

$$
R_{\mu \nu \sigma}^{\alpha} \stackrel{\text { def }}{=}\left\{\begin{array}{c}
\alpha \\
\mu \sigma
\end{array}\right\}_{, \nu}-\left\{\begin{array}{c}
\alpha \\
\mu \nu
\end{array}\right\}_{, \sigma}+\left\{\begin{array}{c}
\epsilon \\
\mu \sigma
\end{array}\right\}\left\{\begin{array}{c}
\alpha \\
\epsilon \nu
\end{array}\right\}-\left\{\begin{array}{c}
\epsilon \\
\mu \nu
\end{array}\right\}\left\{\begin{array}{c}
\alpha \\
\epsilon \sigma
\end{array}\right\},
$$

is the Riemann- Christoffel curvature tensor and the tensor $Q_{\mu \nu \sigma}^{\alpha}$ is defined by

$$
Q_{\mu \nu \sigma}^{\alpha} \stackrel{\text { def }}{=} \gamma_{\substack{\mu \sigma \\++\mid \nu}}^{\alpha}-\gamma_{+-\mid \sigma}^{\alpha}-\gamma_{\mu \sigma}^{\beta} \gamma_{\beta \nu}^{\alpha}+\gamma_{\mu \nu}^{\beta} \gamma_{\beta \sigma}^{\alpha}
$$

where the (-)sign is used to characterize covariant derivatives using the dual connection $\tilde{\Gamma}_{. \mu \nu}^{\alpha}\left(=\Gamma_{. \nu \mu}^{\alpha}\right)$.

Now to get a non-vanishing curvature, we have to parameterize (10) by replacing it with $^{8}$,

$$
\nabla_{. \mu \nu}^{\alpha}=\left\{\begin{array}{c}
\alpha \\
\mu \nu
\end{array}\right\}+b \gamma_{. \mu \nu}^{\alpha},
$$

where $b$ is a dimensionless parameter. The curvature tensor corresponding to (18) can be written as,

$$
\hat{B}_{. \mu \nu \sigma}^{\alpha}=R_{. \mu \nu \sigma}^{\alpha}+b Q_{. \mu \nu \sigma}^{\alpha},
$$

which is, in general, a non-vanishing tensor. The version of the AP-geometry built using (18) is known as the PAP-geometry ${ }^{9}$. The path equations corresponding to (18) can be written as,

$$
\frac{d^{2} x^{\mu}}{d \tau^{2}}+\left\{\begin{array}{c}
\mu \\
\alpha \beta
\end{array}\right\} \frac{d x^{\alpha}}{d \tau} \frac{d x^{\beta}}{d \tau}=-b \quad \Lambda_{\alpha \beta}^{. \mu} \frac{d x^{\alpha}}{d \tau} \frac{d x^{\beta}}{d \tau} .
$$

where $\tau$ is a scalar parameter. It can be easily shown that (18) defines a non-symmetric, metric linear connection. 


\section{Torsion Energy and Energy Densities}

Using equation (15) we can write

$$
R_{. \mu \nu \sigma}^{\alpha} \equiv-Q_{. \mu \nu \sigma}^{\alpha}
$$

Although the tensors $R_{. \mu \nu \sigma}^{\alpha}$ and $Q_{. \mu \nu \sigma}^{\alpha}$ appear to be mathematically equivalent, they have the following differences:

1- The Riemann-Christoffel curvature tensor is made purely from Christoffel symbols

(see (16)), while the tensor $Q_{. \mu \nu \sigma}^{\alpha}(17)$ is made purely from the contortion (11)

( or from the torsion using (13)). The first tensor is non-vanishing in Riemannian

geometry while the second vanishes in the same geometry.

2- The non-vanishing of $R_{. \mu \nu \sigma}^{\alpha}$ is the measure of the curvature of the space, while the addition of $Q_{. \mu \nu \sigma}^{\alpha}$ to it causes the space to be flat. So, one is causing an inverse effect, on the properties of space-time, compared to the other. For this reason

we call $Q_{. \mu \nu \sigma}^{\alpha}$ "The Curvature Inverse of Riemann-Christoffel Tensor" ${ }^{9}$, or "The Additive inverse of the Curvature Tensor". Note that both tensors are

considered as curvature tensor, but one of them cancels the effect of the other, if both existed in the same geometric structure.

Now, in view of the above two differences, we can deduce that these two tensors are not, in general, equivalent. In other words, if we consider gravity as curvature of spacetime and is represented by $R_{. \mu \nu \sigma}^{\alpha}$, we can consider $Q_{. \mu \nu \sigma}^{\alpha}$ as representing anti-gravity! The existence of equal effects of gravity and anti-gravity in the same system neutralizes the space-time, geometrically. This situation is similar to the existence of equal quantities of positive and negative electric charges in the same system, which neutralizes the system electrically.

If we assume that gravity and anti-gravity effects are not exactly equal in the same system, then space-time curvature can be represented by the tensor (19). The existence of anti-gravity gives rise to a repulsive force, which can be used to interpret SN type Ia observation. This can be achieved by adjusting the parameter $b$. The above discussion gives an argument on the production of a repulsive force by torsion.

It is well known that the L.H.S. of (21) satisfies the second Bianchi Identity, so using (21) we can easily show that ${ }^{10}$

$$
\sum_{; \beta}^{\alpha \beta} \equiv 0
$$

where,

$$
\begin{gathered}
\Sigma^{\alpha \beta} \stackrel{\text { def }}{=} Q^{\alpha \beta}-\frac{1}{2} g^{\alpha \beta} Q, \\
Q_{\alpha \beta} \stackrel{\text { def }}{=} Q^{\sigma}{ }_{. \alpha \beta \sigma} \\
Q \stackrel{\text { def }}{=} g^{\alpha \beta} Q_{\alpha \beta} .
\end{gathered}
$$

It is clear from (22) that the physical quantity represented by the tensor $\Sigma^{\alpha \beta}$ is a conserved quantity. We are going to call it the "Torsion Energy", since $Q_{. \mu \nu \sigma}^{\alpha}$ is purely made of the torsion as mentioned above (see (17)). 
As stated above, curvature and torsion energies are defined by (2) and (23), respectively. We can take the norm of the scalers $G, \Sigma$ to represent the curvature and torsion energy densities, respectively. Both have the dimensions $\mathrm{cm}^{-2}$ characterizing energy density, in relativistic units. Now the total energy of a system with curvature and torsion can be written as:

$$
E=\|G\|+\|\Sigma\|
$$

where,

$$
\Sigma \stackrel{\text { def }}{=} g_{\mu \nu} \Sigma^{\mu \nu}
$$

and

$$
G \stackrel{\text { def }}{=} g_{\mu \nu} G^{\mu \nu}
$$

We can define the following parameters:

$$
\begin{array}{ll}
\text { Curvature energy density parameter : } & \Omega_{c r} \stackrel{\text { def }}{=} \frac{\|G\|}{E}, \\
\text { Torsion energy density parameter : } & \Omega_{t r} \stackrel{\text { def }}{=} \frac{\|\Sigma\|}{E} .
\end{array}
$$

Then equation (26) can be written as,

$$
1=\Omega_{c r}+\Omega_{t r}
$$

If we assume that (29) represents the total energy parameter collecting different sources of energy causing curvature and torsion of space-time, and dark energy is due to torsion of space time, then we can take ${ }^{11} \Omega_{t r}=\frac{2}{3}$. This will put a limit on the parameter $b(\simeq 2)$ and consequently on the parameter $\gamma(\simeq 548)$. These limits are calculated assuming that the material constituents of the Universe are almost fermions. Note that the value of the parameter $\gamma$ is unity in the case of the Earth's gravitational field ${ }^{12}$. It seems that its value depends on the size of the system under consideration.

As a second argument on the existence of a repulsive force, corresponding to the torsion of space-time, consider the linearized form of (20) which can be written $\mathrm{as}^{8}$,

$$
\Phi_{T}=\Phi_{N}(1-b)=\Phi_{N}+\Phi_{\Sigma}
$$

where,

$$
\Phi_{\Sigma} \stackrel{\text { def }}{=}-b \Phi_{N}
$$

$\Phi_{N}$ is the Newtonian gravitational potential and $\Phi_{T}$ is the total gravitational potential due the presence of gravity and anti-gravity. It is clear from (30) that the Newtonian potential is reduced by a factor $b$ due to the existence of the torsion energy. It is obvious from (31) that $\Phi_{\Sigma}$ and the Newtonian potential have opposite signs. Then one can deduce that $\Phi_{\Sigma}$ is a repulsive gravitational potential, in contrast to the attractive gravitational potential $\Phi_{N}$. 


\section{Discussion and Concluding Remarks}

In the present work we have chosen a version of the 4-dimensional AP-geometry to represent the physical world including space and time. This version, the PAP-geometry, is more wider than the Riemannian geometry, since it has simultaneously non-vanishing curvature and torsion. The PAP-geometry is characterized by the parameter $b$. If $b=0$, this geometry becomes Riemannian, while if $b=1$, it recovers the conventional AP-geometry. On the present work, we can draw the following remarks:

1- We have applied the geometrization philosophy and its guide lines mentioned in section 2 , to a geometry with curvature and torsion. One can summarize the results of this application in the following points, corresponding to the three guide lines given in section 2.

(i) A conservation law, as a law of nature, giving conservation of torsion energy is represented by the differential identity (22).

(ii) The quantity called the "torsion energy" is represented by the tensor (23), which is a part of a geometric structure. This tensor gives rise to anti-gravity and consequently a repulsive force.

(iii) Trajectories of test particles affected by the torsion of the space-time is represented by the path equation (20), which is a curve in the geometric structure used.

The path equation (20) has been used to study trajectories of spinning elementary test particles in a background with torsion and curvature. The R.H.S. of this equation is suggested to represent a type of interaction between torsion and the quantum spin of the moving particle ${ }^{8}$. The application of this equation to the motion of the thermal neutrons in the Earth's gravitational field removes the discrepancy in the COW-experiment ${ }^{12}$.

2- It is clear that torsion energy, defined in section 4., can solve the problem of SN type Ia observations, since it gives rise to a repulsive force. This can be achieved by adjusting the parameter $b$, as shown in section 4 . One can now replace the exotic term "dark energy" by the term "torsion energy". The later has a known origin (a pure geometric origin). This shows the success of the geometrization scheme in dealing with physical problems.

3- Both terms "dark energy" and "cosmological constants" are exotic terms. They have neither geometric origin nor well defined physical origin.

4- Many authors consider $R_{. \mu \nu \sigma}^{\alpha}$ and $Q_{. \mu \nu \sigma}^{\alpha}$ as identical tensors from both physical and mathematical points of view. For this reason, they are using the term Teleparallel equivalent of $G R$ for theories built using $Q_{. \mu \nu \sigma}^{\alpha}$. In view of the present work, such authors are, in fact, constructing anti-gravity theories. (This is similar to the situation of constructing a field theory for positive electric charges and another one for negative electric charges. Such theories would be similar). Discrepancies would appear only if both fields existed in the same system, as in the case of SN type Ia observations.

5- Curvature and torsion corresponds to two different types of energy. The energy 
corresponding to the first gives rise to an attractive force, while the energy corresponding to the second is repulsive. We believe that torsion energy is what has been discovered recently by the SN type Ia observation ${ }^{1}$. Both energies have the same conservation law.

6- In the context of the present work, we can extend the geometrization philosophy by adding the following postulate.

Physical phenomenae are just interactions between the space-time structure and

the intrinsic properties of the material constituents of the system.

Using this postulate, the attractive force is interpreted as a result of the interaction between the curvature of space-time and the mass (energy) of the material constituents; while the repulsive force can be interpreted as a result of an interaction between the torsion and the quantum spin of the material constituents 8 .

7- A successful description of gravity is to be given using a more complete geometry, i.e. a geometry with a simultaneously non-vanishing curvature and torsion.

8- The parameter $\gamma$ is a dimensionless parameter. It is suggested to be fixed by experiment or observation ${ }^{8}$. This parameter characterizes the source of the background field. It has been shown that $\gamma=1$ in the case of the Earth as a source of the gravitational field in the COW-experiment ${ }^{12}$. As shown in section $4, \gamma=548$ for the Universe. This parameter is system dependent. The type of dependence of $\gamma$ can be understood in the context of a field theory constructed in the PAP-geometry.

9- The results obtained in the present work can be obtained, with some efforts, using other geometries with curvature and torsion, since such geometries possess similar features ${ }^{13}$.

\section{Acknowledgment}

The author would like to thank the organizing committee of the conference and Dr. S. Khalil for inviting him to give this talk.

\section{References}

1. J.L. Tonry, B.P. Schmdit, et al. Astrophys. J.594, 1 (2003).

2. P.D. Mannheim, Prog.Part.Nucl.Phys. 56340 (2006); gr-qc/0505266

3. S.M. Carroll, "The Cosmological Constant", http://livingreviews.org/ Irr-2001-1.

4. A. Einstein, "The Meaning of Relativity" (Princeton, 5th ed. 1955).

5. A. Einstein, Math. Annal. 102, 685 (1930).

6. M.I. Wanas, Cercet.Stiin.Ser.Mat. 10, 297 (2001); gr-qc/0209050.

7. K. Hayashi and T. Shirafuji, T. Phys.Rev. D19, 3524 (1979).

8. M.I. Wanas, Astrophys. Space Sci., 258, 237 (1998); gr-qc/9904019

9. M.I.Wanas, Turk. J. Phys. 24, 473 (2000); gr-qc/0010099

10. M.I. Wanas, arXiv:0705.2255 (2007).

11. W.L.Friedman and M.S. Turner, Rev.Mod.Phys. 751433 (2003). 
12. M.I.Wanas, M. Melek and M.E. Kahil, Gravit. Cosmol.,6, 319 (2000).

13. M.I. Wanas and M.E. Kahil, Gen.Rel.Gravit. 311921 (1999); arXiv:9912007. 